\title{
Le paracétamol, de nouvelles cibles pour un vieux médicament
}

\section{Paracetamol: new targets for an old drug}

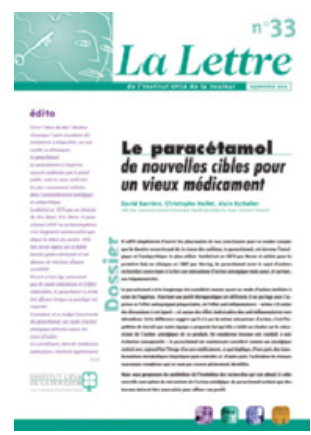

Fièvre ? Maux de tête ? Douleur chronique ? Après inventaire des traitements à disposition, un seul semble se démarquer : le paracétamol.

Synthétisé en 1878 par un chimiste de chez Bayer, H.N. Morse, il n'est largement commercialisé que depuis le début des années 1950.
Malgré l'ancienneté du paracétamol, son mode d'action antalgique présente encore des zones d'ombre que les chercheurs tentent d'éclaircir.

Réalisée en collaboration avec David Barrière, Bernard Calvino, Alain Eschalier et Christophe Mallet, cette $33^{\mathrm{e}}$ édition de La lettre de l'institut UPSA de la douleur se penche sur ce « vieux » médicament qui a encore de l'avenir et qu'on ne cesse de redécouvrir.

Les auteurs qui réalisent dans cette lettre une synthèse de l'évolution des recherches ayant abouti à cette nouvelle conception du mécanisme de l'action antalgique du paracétamol déclarent que « de nombreux travaux ont conduit à une évolution conceptuelle : le paracétamol étant considéré comme un antalgique central avec aujourd'hui l'image d'un promédicament, ce qui implique, d'une part des transformations métaboliques hépatiques puis centrales et, d'autre part, l'activation de réseaux neuronaux complexes qui ne sont pas encore pleinement identifiés »".

Cette lettre est disponible sur le site www.institut-upsadouleur.org. 